\title{
Informes
}

\section{Brígida García Guzmán: Profesora-Investigadora Emérita de El Colegio de México}

\author{
Brígida García Guzmán: \\ Research Fellow Emerita at El Colegio de México
}

\begin{abstract}
Resumen
La doctora Brígida García Guzmán recibió la distinción Profesora-Investigadora Emérita de El Colegio de México en reconocimiento a su destacada trayectoria académica en el área de la sociodemografía, así como por su comprometida labor en la formación de estudiosos de la población. La ceremonia se llevó a cabo el 24 de junio de 2019 en la Sala Alfonso Reyes de dicha institución.

A continuación, presentamos las palabras de los oradores en el orden de su intervención: Silvia E. Giorguli, presidenta de El Colegio de México; Orlandina de Oliveira, Profesora-Investigadora Emérita de la misma institución; Enrique de la Garza Toledo, Profesor Distinguido de la Universidad Autónoma Metropolitana; y Jessica Nájera, profesora-investigadora del Centro de Estudios Demográficos, Urbanos y Ambientales de El Colegio. Asimismo, incluimos las palabras de Brígida García Guzmán, ahora Profesora-Investigadora Emérita de El Colegio de México. Fungió como moderador Vicente Ugalde, secretario académico de esa institución.
\end{abstract}

\begin{abstract}
Dr. Brigida del Carmen Garcia Guzmán received the Research Fellow Emerita distinction from El Colegio de México in recognition of her outstanding academic career in the area of sociodemography, and her committed work in the training of population scholars. The ceremony was held on June 24, 2019, in the Alfonso Reyes Hall of this institution.

The words of the speakers are reproduced below in order of appearance: Silvia E. Giorguli, president of El Colegio de México; Orlandina de Oliveira, Research Fellow Emerita of the same institution; Enrique de la Garza Toledo, Distinguished Professor of the Autonomous Metropolitan University; and Jessica Nájera, research fellow at the Center for Demographic, Urban and Environmental Studies of El Colegio. We also include the words of Brigida Garcia Guzmán, now Research Fellow Emerita of El Colegio de México. Vicente Ugalde, academic secretary of that institution, served as moderator.
\end{abstract}




\section{Palabras de Silvia E. Giorguli*}

Bienvenidos todos a El Colegio de México. Es un placer observar la asidua asistencia a esta ceremonia dedicada a reconocer la larga y destacada trayectoria de la doctora Brígida García Guzmán. Recibimos con gusto a muchas generaciones de demógrafos -desde las primeras hasta las más recientes-, a los estudiantes actuales, a los egresados que viajaron desde fuera de la Ciudad de México y también a los que vienen desde el extranjero para acompañarnos el día de hoy. También es gratificante observar la presencia de colegas y egresados de otros centros, así como de las compañeras secretarias que han colaborado con ella a lo largo de estas cuatro décadas. Finalmente, nos honra recibir a la familia de Brígida, a su esposo -el doctor Gustavo Garza- y a sus hijos, quienes no podían faltar en este festejo.

En estas palabras de bienvenida a la ceremonia que hoy nos convoca, no voy a hacer una intervención detallada sobre la larga trayectoria de la doctora García porque sé que quienes me acompañan en el presídium lo harán con mayor detalle y conocimiento de sus diversas aportaciones. Aun así, no podía dejar pasar la ocasión para, de mi parte y como presidenta de la institución, hacer una mención reconociendo las varias facetas y contribuciones de Brígida García como profesora-investigadora en El Colegio de México y en el campo de la sociodemografía.

\section{Brígida García, una demógrafa pionera en el campo del trabajo femenino en México}

Probablemente uno de los rasgos que más destacan de la carrera académica de Brígida es su aportación, en colaboración con otros miembros de este claustro y de otras instituciones, para la construcción de una agenda de investigación en torno a los estudios de la familia, que integra de manera sistemática y con un estudio riguroso el análisis de la participación de las mujeres en el mercado de trabajo en México. De alguna manera, la división tradicional del trabajo en el país, los estigmas sobre los roles de las mujeres y de los hombres, así como la baja participación de ellas en el trabajo formal, hacían invisible la presencia y las aportaciones de las mujeres con su trabajo-doméstico y extradoméstico-a los hogares en México. Brígida es, junto con otros varios colegas que hoy nos acompañan, pionera en la visibilización

\footnotetext{
* Presidenta de El Colegio de México,A.C. Correo: sgiorguli@colmex.mx ORCID: https:// orcid.org/0000-0003-4573-9389
} 
del trabajo femenino y en la construcción de una agenda de investigación que habría de desarrollarse con mayor fuerza desde los ochenta. La apertura sistemática de este campo de investigación conllevó, además, la innovación de ubicar la discusión en la intersección entre tres líneas de análisis: la familia, los mercados de trabajo (el trabajo femenino en específico) y la dimensión de género.

Hay varias particularidades en la participación de la doctora García en esta intersección, que es, a final de cuentas, una propuesta analítica para estudiar el trabajo femenino, sus vinculaciones con otros procesos y sus implicaciones en diversas dimensiones, incluida la de las desigualdades de género. La construcción de esta agenda de investigación ocurrió paralela a la formación de diversas generaciones de demógrafos que pasamos por estas aulas y que pudimos discutir con Brígida García sobre dicho tema. El enfoque de la profesora conlleva e invita a un balance entre una discusión teórica estricta que combina las aportaciones desde la sociología, la economía laboral y los estudios de género con la reflexión sobre las aproximaciones metodológicas y la generación de las estrategias y los datos necesarios para desarrollar la investigación. De ahí que cualquiera interesado en seguir la trayectoria de Brígida en sus publicaciones y en las tesis que ha dirigido, encontrará contribuciones tanto en lo teórico como en la presentación de diagnósticos del momento y sobre el cuidado en el manejo del dato. Creo que este rasgo -la rigurosidad y sistematicidad en la investigación- ha sido y sigue siendo uno de los distintivos en la forma de investigar en El Colegio de México, no sólo en el aspecto sociodemográfico, sino también entre diversas disciplinas y generaciones.

\section{Las contribuciones en la generación de información para estudiar el trabajo femenino en México}

En esta misma línea de argumentación hay un aspecto del trabajo de Brígida García que conozco desde que me incorporé a El Colegio de México y al gremio demográfico. Me refiero a la preocupación por el dato. Si uno busca en la historia de los censos y de las encuestas y estadísticas de empleo, va a encontrar el énfasis de Brígida y de otros colegas de este campo del conocimiento sobre la necesidad de analizar el dato, mejorarlo y captar de la mejor manera posible el trabajo femenino en México. No es un tema menor porque, entre los asuntos que resaltan y que han visibilizado Brígida y los colegas es que, según la forma en que se pregunte, se obtendrán respuestas diferentes acerca de lo que las mujeres entienden sobre su participación en el trabajo 
extradoméstico. Esta línea de acción dentro de la investigación ha llevado a que se desarrolle una interlocución -a veces amable y otras ríspida-con los generadores de información, principalmente con el Instituto Nacional de Geografía y Estadística (INEGI). Por supuesto que esta misma discusión se da, con matices diversos, respecto de otros ámbitos de la información sociodemográfica. En el caso de Brígida y de varios de sus colegas cercanos, se trata, de nuevo, de abrir campo para captar en las estadísticas algo que no se veía como relevante medir, y en mantener a México en la vanguardia en la generación de información sobre el trabajo femenino. La intersección con otros aspectos, como el de la formación familiar o la violencia doméstica, ubican una parte de esta discusión sobre el dato en los avances que ha habido en el país en torno a las estadísticas de género.

En resumen, para cerrar esta reflexión, desde la parte académica, Brígida García nos ha transmitido a las diversas generaciones que nos hemos formado después de ella, hasta la más reciente, una forma de hacer investigación con pasión, con gran apertura para compartir y con gran cuidado metodológico. Se trata además de una agenda de investigación que se ha renovado, que se sigue actualizando y que ahora abarca temas como el uso del tiempo y la medición de las contribuciones del trabajo doméstico, como ejemplos.

\section{Brígida García, la profesora formadora de varias generaciones de demógrafos}

Quiero también aprovechar este espacio para hacer un reconocimiento a Brígida la profesora, la formadora de formadores, la directora de tesis, la participante en múltiples comités dentro y fuera de la institución, la mentora generosa para compartir conocimiento y también preocupada por transmitir una forma de hacer investigación. Los ejemplos y testimonios son múltiples, como los de varios de sus estudiantes que nos acompañan el día de hoy. Son demógrafos que, ahora desde sus instituciones, siguen desarrollando agendas de investigación que se derivan de su trabajo de tesis original, $\mathrm{y}$ creo que esa es la mejor manera de honrar las enseñanzas de una profesora como Brígida, y reconocer el apoyo que ha dado, y que sigue dando, a los investigadores jóvenes que ha dirigido y formado. 


\section{La construcción institucional}

Este es el espacio para también reconocer la participación de la doctora Brígida García en la vida y la construcción institucional del CEDUA y de El Colegio. Cuando uno revisa el pasado, advertimos que Brígida fue integrante de todos los órganos colegiados de nuestra institución, ¡de todos! Fue miembro de la Junta de Gobierno, del Consejo Académico y de la Comisión Dictaminadora. Dentro del CEDUA participó en las discusiones de los programas docentes y de las líneas de investigación. Destacó en particular su liderazgo, conjuntamente con Susana Lerner y otros colegas del Centro, en el diseño y apertura del doctorado en Estudios de Población. En cuanto a sus líneas de investigación, después de que me incorporé a la planta académica del CEDUA tuve la oportunidad de participar con Brígida en la construcción y consolidación de la línea trabajo, familia y género, donde existía una intersección y un componente con el tema de migración.

La participación de Brígida no se restringe a El Colegio de México. En el gremio de los demógrafos son bien conocidas y reconocidas sus contribuciones desde la Sociedad Mexicana de Demografía, de la cual fue presidenta. Fue además una de las impulsoras en la creación de la Asociación Latinoamericana de Población y en la formación de redes y colaboraciones académicas con otras instituciones en la región, en particular en los temas de familia y trabajo. Su actividad dentro del gremio se mantiene y sigue colaborando con la apertura de la agenda de investigación en temáticas como el uso del tiempo, lo cual nos hace esperar su activa participación en los futuros foros regionales de discusión.

\section{Un agradecimiento por muchas razones}

Quisiera cerrar mis palabras desde un comentario más personal y hablar de lo que Brígida ha representado en mi trayectoria. La ceremonia de hoy es, a fin de cuentas, también una oportunidad para agradecer. Yo tuve la fortuna de tener en mi comité de tesis doctoral a dos grandes demógrafas: Frances Goldscheider y Brígida García. Dado que me encontraba terminando mi doctorado en Estados Unidos, la retroalimentación de Brígida a mi trabajo fue fundamental. Siempre fue un referente por la investigación que ella había desarrollado hasta ese momento, pero también sus comentarios fueron indispensables para aterrizar mi investigación en el contexto de México e incorporar sus particularidades. Se convirtió en mi referente de la discusión teórica que se daba en el país y en América Latina y me dio elementos para 
interpretar los resultados desde nuestra propia narrativa. Le agradezco hoy a Brígida su generosidad por el tiempo que ocupó para leer mi trabajo, así como por comentar, discutir y criticar con mucho detalle todo el conjunto de la tesis.

Además, quiero agradecerle a Brígida el acompañamiento y las conversaciones en más de un momento de mi trayectoria: desde mi ingreso a El Colegio de México, en torno a El Colegio como un "proyecto institucional" cuando decidí participar en el proceso de nombramiento de la dirección del CEDUA, como presidenta y vicepresidenta de Somede, entre otros. Le agradezco el apoyo que me dio en el impulso a proyectos nuevos, que ahora vemos más consolidados, como la revista Coyuntura Demográfica. Desde que planteamos la iniciativa, Brígida nos apoyó promoviéndola, empujándola y regalándonos su tiempo, sobre todo en la primera etapa. Finalmente, quiero agradecerle los espacios para discutir sobre todo este proyecto institucional que ahora encabezo en la Presidencia de El Colegio.

Brígida, ¡muchísimas gracias por todo! ¡Felicidades por el merecido reconocimiento a tu trayectoria académica, a tu compromiso y visión en tu trabajo de investigación, y a la formación de muchas generaciones aquí en El Colegio de México! 


\section{Palabras de Orlandina de Oliveira*}

Distinguidos miembros del presídium, colegas y amigos que nos acompañan:

Agradezco la invitación a decir estas palabras y me honra participar en la ceremonia en la que se otorga a Brígida García el nombramiento de Profesora-Investigadora Emérita de El Colegio de México. En mi intervención, busco ofrecerles un breve panorama de su ejemplar trayectoria académica

Brígida García se ha distinguido por impulsar de manera pionera el desarrollo del campo de la sociodemografía en el país; su liderazgo ha sido indiscutible y su presencia en la esfera internacional es considerable. En el ámbito de la investigación, sus contribuciones abarcan varias áreas de estudio: mercados laborales, familia, trabajo no remunerado y desigualdades de género.

Considero de primer orden reconocer que su práctica de investigación en equipos de trabajo o en forma individual ha sido creativa, sistemática y rigurosa, y ha llevado a producir conocimiento original sobre aspectos de la realidad social poco estudiados en su momento histórico.

Las estrategias analíticas que utiliza son complejas, pues combinan el uso de un marco conceptual crítico con el manejo de datos cuantitativos y cualitativos, así como de información transversal y longitudinal. Su perspectiva ha sido esencialmente interdisciplinaria y armoniza elementos teóricos y metodológicos de la demografía y la sociología.

En diferentes etapas de su carrera profesional ha hecho relevantes aportes al estudio de los cambios ocurridos en los mercados de trabajo en el ámbito nacional y en el regional, así como en las grandes ciudades del país. Su interés central ha sido profundizar en las repercusiones de las transformaciones económicas en la esfera laboral.

Sus primeros análisis fueron sobre la expansión o contracción de los distintos sectores de trabajadores asalariados y no asalariados. Brígida constató que el proceso de salarización de la mano de obra no avanzaba en México al ritmo que lo hizo en los países desarrollados, y que éste no era un proceso lineal: ocurrían avances y retrocesos según el momento histórico analizado. Esta investigación culminó con la publicación del libro Desarrollo económico y absorción de fuerza de trabajo en México, 1950-1980.

Destacan, asimismo, sus estudios acerca de los cambios en el trabajo femenino durante distintos periodos, y en especial sobre lo que ocurrió durante la crisis de los años 1980, cuando comenzó el ajuste y la reestructuración

* Profesora-Investigadora Emérita de El Colegio de México, A.C. Correo: odeolive@ colmex.mx ORCID: https://orcid.org/0000-0001-6882-4346 
económica del país. Ellos permitieron documentar la transformación en el perfil de la mano de obra femenina en esos años, y destacaron la importante presencia de mujeres casadas y de mayor edad en el mercado de trabajo, quienes buscaban de esa manera contribuir a la subsistencia de sus hogares.

Esta línea de estudio también ha permitido precisar las diferentes facetas de la heterogeneidad laboral que caracteriza tanto a la mano de obra masculina como femenina en México, así como las distintas manifestaciones del deterioro en las condiciones de trabajo, en términos de bajos salarios, ausencia de prestaciones sociales y utilización de contratos verbales o temporales.

En el campo de estudio sobre hogares y familias, una de sus primeras inquietudes fue investigar la participación económica de la mano de obra familiar como parte de las estrategias a las que recurren los individuos y las familias de diferentes sectores sociales para mantener su nivel de vida. Se analizó la presencia económica masculina y femenina considerando el hogar como unidad de análisis, y se utilizó el concepto de "contextos familiares" para examinar cómo dicha presencia variaba de acuerdo con el tipo de hogar, la etapa del ciclo familiar y la inserción laboral del jefe.

Posteriormente, su interés se orientó hacia la investigación de la influencia del incremento del trabajo femenino sobre la condición social de las mujeres en diferentes contextos urbanos (Ciudad de México, Mérida y Tijuana). Se trataba de examinar el trabajo femenino desde ópticas diversas: su expansión, sus condicionantes, su repercusión sobre la autonomía femenina y su significado en diferentes sectores sociales y contextos urbanos. Aquí el reto principal era no sólo ver las vinculaciones entre diferentes niveles de análisis, sino también incorporar las concepciones de las propias mujeres sobre el trabajo, la maternidad y las relaciones de género.

Años más tarde, buscó profundizar en el entendimiento de la dinámica familiar y sus consecuencias sobre la condición social de las mujeres. Las formas de convivencia familiar han recibido una atención especial mediante el análisis de los patrones de autoridad, libertad de movimiento y violencia doméstica. Con base en las visiones de las mujeres y de los varones, analizó situaciones y aspectos que propician relaciones familiares más igualitarias.

Recientemente, Brígida se ha centrado en el estudio del uso del tiempo y el trabajo no remunerado doméstico y de cuidado en México. En su más reciente artículo, sistematiza y evalúa los avances logrados en diferentes aspectos sobre este tema, así como también plantea los retos que enfrenta la investigación sociodemográfica en torno al mismo.

Su contribución a la formación de recursos humanos ha sido, de igual forma, destacada. Como docente, su compromiso con las nuevas generacio- 
nes de demógrafos ha sido innegable; su labor siempre ha sido de una gran seriedad y responsabilidad. Mediante la impartición de cursos y seminarios de metodología, fuerza de trabajo y familia, la dirección de tesis y la participación en comisiones lectoras, ha contribuido a la formación de investigadores con conocimientos sólidos, rigor científico y sentido crítico.

A la par de su actividad como investigadora y docente, Brígida, a lo largo de su carrera académica, ha contribuido de manera importante al desarrollo institucional al participar en un buen número de órganos colegiados de gestión y evaluación, así como en esfuerzos editoriales de distinta índole y en la elaboración de programas docentes. Mención particular merecen las actividades de gestión desarrolladas en El Colegio. En nuestra institución, ha sido parte de juntas de profesores, del Consejo Académico y de varias otras comisiones y cuerpos colegiados. Fue la primera directora de la revista Estudios Demográficos y Urbanos. Sobresale su gestión como miembro de la Comisión Dictaminadora General y de la Junta de Gobierno de El Colegio. Asimismo, fue defensora de los derechos de la comunidad de nuestra institución de 2015 a 2018. Vale mencionar, también, que fue integrante de la Junta de Gobierno de El Colegio de Sonora.

Resalta, de igual forma, su colaboración en comisiones dictaminadoras o en otras instancias de gestión y evaluación en varias instituciones de investigación y docencia en el país. Entre otras, en el Instituto de Investigaciones Sociales y el Centro Regional de Investigaciones Multidisciplinarias de la UNAM, la Facultad Latinoamericana de Ciencias Sociales, El Colegio de la Frontera Norte, El Colegio Mexiquense y la Universidad Autónoma del Estado de Morelos. Pero sin duda, su labor más demandante, en cuanto a la evaluación de sus pares, fue en la Comisión de Ciencias Sociales del Sistema Nacional de Investigadores (SNI), la cual presidió en 2004.

Como reconocimiento a su trayectoria académica, Brígida ha sido acreedora de varias distinciones. Cabe mencionar: el Premio Luisa María Leal Duk en 2003 por parte del Foro Nacional de Mujeres y Políticas de Población; el homenaje por su notoria contribución a la demografía por parte de la Sociedad Mexicana de Demografía (Somede) en 2010; y el nombramiento de Investigadora Emérita del Sistema Nacional de Investigadores (SIN) en 2015.

Asimismo, ha recibido importantes apoyos internacionales para su actividad profesional por parte del Population Council y de las fundaciones Ford, Rockefeller y MacArthur, así como de la Organización Mundial de la Salud, el Fondo de Población de las Naciones Unidas (UNFPA) y ONUMujeres, entre otras organizaciones.

Igualmente significativas han sido las invitaciones para participar como visitante en centros de investigación de Brasil, Estados Unidos, España e 
Inglaterra, entre los que están: el Centro Brasileño de Análisis y Planeación (Cebrap) en São Paulo, la Universidad de California en San Diego, la Universidad de Texas en Austin, la Universidad John Hopkins en Maryland, la Universidad Complutense de Madrid y la Universidad de Essex en Inglaterra.

Por último, quiero enfatizar su labor de liderazgo en los estudios de población señalando su activa presencia en organizaciones nacionales e internacionales. En la Somede se ha distinguido por formar parte de comités de gestión y promoción de su campo disciplinario desde su fundación en 1981. Cuando fue presidenta de esta importante asociación de demografía (1994-1996), formó parte de la Delegación Oficial de México en la Conferencia de Beijing e impulsó la presencia de un grupo de demógrafos mexicanos en la sección no gubernamental.

Desde ese entonces, ha buscado por diversos medios ampliar la presencia de los demógrafos en la sociedad mexicana; en especial, cabe destacar su participación en la Contraloría Social del Programa Nacional de la Mujer (el órgano que precedió al Instituto Nacional de las Mujeres) y en el Consejo Consultivo Ciudadano para la Política de Población, en la Secretaría de Gobernación.

En el ámbito internacional, en los años 1970 y 1980 formó parte activa de la Comisión de Población y Desarrollo del Consejo Latinoamericano de Ciencias Sociales, y del Programa Latinoamericano de Actividades en Población (Prolap), grupos pioneros dentro del Consejo Latinoamericano de Ciencias Sociales (Clacso) que impulsaron una redefinición del campo de los estudios demográficos, otorgando un papel primordial a la desigualdad social en la comprensión del elevado crecimiento demográfico que prevalecía en ese entonces. Dichos grupos fueron precursores de lo que es hoy la Asociación Latinoamericana de Población (ALAP).

En lo que respecta a la Unión Internacional para el Estudio Científico de la Población (IUSSP), durante los años noventa su presencia fue activa. Como miembro del Comité sobre Género y Población, que convocaba académicos de muy diferentes latitudes, coordinó la publicación de dos libros: Women, poverty and demographic change y Women in the labor market in changing economies, ambos publicados por Oxford University Press. Estas publicaciones tratan temas y estrategias metodológicas novedosos y requirieron la conformación de equipos de trabajo internacionales.

Sin lugar a duda, la trayectoria de Brígida ha sido y sigue siendo en muchos sentidos excepcional; se trata de una vida profesional dedicada enteramente a la investigación, a la formación de recursos humanos y a la divulgación del quehacer científico. 
Concluyo resaltando su gran valor como ser humano, su capacidad, integridad, generosidad, responsabilidad y respeto por los demás. Para mí, ha sido un privilegio contar con su amistad incondicional. No puedo dejar de mencionar lo enriquecedora que ha sido la experiencia de trabajar en equipo con Brígida a lo largo de más de cuarenta años de nuestra vida profesional. Me permito felicitar a nuestra nueva Profesora-Investigadora Emérita de El Colegio de México. 


\section{Palabras de Enrique de la Garza Toledo*}

Me siento muy honrado de que me hayan invitado a dirigirles unas breves palabras con motivo de haberse otorgado la distinción de Profesora-Investigadora Emérita a la doctora Brígida García.

Los méritos académicos de Brígida son innegables. No quisiera hacer una larga contabilidad de su producción académica, pero no puedo dejar de mencionar que obtuvo el doctorado en Sociología en la Facultad de Ciencias Políticas y Sociales de la UNAM, y que muy pronto se incorporó a El Colegio de México, donde desde 1971 ha desarrollado su vida como docente e investigadora.

Ha recibido gran cantidad de distinciones; entre ellas cabe destacar el nivel III del Sistema Nacional de Investigadores (SNI) y el de Investigadora Emérita por la misma institución.

Ha publicado 13 libros, 56 capítulos de libro y 51 artículos; ha dirigido 15 tesis de doctorado y 22 de maestría, todas concluidas. Ha fungido como ponente, coordinadora u organizadora en 195 congresos. Es decir, se trata de una investigadora entregada de por vida a su profesión, que ha tenido importantes productos científicos.

Pero Brígida no sólo ha sido notable en lo cuantitativo de la producción académica, sino que creó, junto con Orlandina de Oliveira y Fernando Cortés, lo que en el Cono Sur conocen como Escuela Mexicana de Sociodemografía del Mercado de Trabajo. En nuestro medio es necesario enfatizar tal reconocimiento en la formación de una escuela de pensamiento teórico -acompañada de importantes investigaciones empíricas-. A pesar de que se diga que nadie es profeta en su tierra, tengo la propuesta para el Colmex de realizar un seminario donde se analicen los aportes de dicha escuela de pensamiento.

Esta escuela, constituida a partir de los años setenta del siglo XX, ahora aparece como "naturalizada", oscureciendo con esto sus orígenes y autores principales. En su tiempo significó una ruptura con las doctrinas dominantes en cuanto a los mercados de trabajo, en especial con aquella que planteaba que la oferta crea su propia demanda.

Tal ruptura significó que en el análisis de la unidad doméstica se abordaría la ocupación con una reformulación del concepto de mercado de trabajo, que incluye las decisiones en la familia respecto a qué miembros lanzar al mercado de trabajo; asimismo, se enfatiza la importancia de las redes sociales para conseguir empleo.

* Profesor Distinguido de la Universidad Autónoma Metropolitana. Correo: egt@xanum. uam.mx ORCID: http://orcid.org/0000-0001-7254-9658 
Dichos planteamientos teóricos se vieron enriquecidos por las investigaciones empíricas, las cuales manejaban muestras representativas de gran volumen, como la fundacional de Hogares y Trabajadores en la Ciudad de México.

En varias ocasiones, algunos que cultivamos el enfoque de los procesos de trabajo, buscamos el acercamiento con esta corriente sociodemográfica. Los resultados fueron varios seminarios de discusión y libros colectivos. Habría que hacer más esfuerzos en este sentido, porque el fenómeno laboral implica varios espacios articulados: el del mercado de trabajo, el del proceso de trabajo y el de las relaciones laborales.

Pero un emérito no es únicamente un investigador muy productivo cuantitativamente, ni tampoco sólo el que plantea una nueva corriente de pensamiento, ni el que exclusivamente ha hecho grandes investigaciones empíricas: debe implicar, también, alguien que con gran pasión ha hecho de la investigación uno de los ejes vitales fundamentales, alguien que, a pesar de su nivel académico, muestra gran tolerancia y generosidad frente a otras perspectivas de análisis; así como un investigador formador de nuevas generaciones

Todo esto ha sido también la vida de Brígida García. Por todo esto, como alumno indirecto de la homenajeada, como amigo y como colega, felicito a Brígida García por haber sido nombrada Profesora-Investigadora Emérita de la institución madre de las ciencias sociales modernas, que nos formó, nos enseñó el rigor en la investigación, a la vez que nos inculcó un espíritu crítico: El Colegio de México. 


\section{Palabras de Jéssica Nájera}

A lo largo de cuatro décadas en El Colegio de México, la doctora Brígida García ha sido una socióloga y demógrafa activa, reflexiva y, sin duda, precursora de la perspectiva sociodemográfica en temas laborales, de familia y género en el país y en América Latina. En este reconocimiento, quiero destacar su aporte como docente desde la experiencia de los que hemos sido sus estudiantes, tesistas y colegas.

Brígida inició su trayectoria como profesora-investigadora en el Centro de Estudios Demográficos Urbanos y Ambientales (CEDUA) en 1971; ha sido profesora de veinte generaciones de estudiantes de la maestría en Demografía (la primera de ellas en 1971); así como profesora de doce generaciones del doctorado en Estudios de Población, a partir de 1985.

Sus cursos impartidos en estos programas docentes convergen en temas como: fuerza de trabajo, fecundidad, familia y género, enfoques teóricos y problemas de los estudios de población (curso troncal del doctorado), desigualdad, pobreza, sociedad, metodología de la investigación en población, cambio social y mercados de trabajo, por mencionar algunos. Como parte de su labor docente, Brígida se distingue por la preocupación constante en el mejoramiento y actualización de los programas docentes del CEDUA, procurando siempre combinar el aporte histórico con los temas poblacionales de vanguardia y la inclusión de nuevas metodologías de investigación.

En las clases, Brígida García nos enseñó a estudiar los lazos entre los miembros de la familia y su grupo social, entre las familias y el mercado laboral, y finalmente, entre los mercados laborales y la participación de la población, cerrando con ello el vínculo entre lo micro, lo meso y lo macrosocial. Nos impulsó a identificar las profundas desigualdades que priman en nuestra sociedad, reflejo de las desigualdades entre las familias y dentro de ellas.

Constantemente, Brígida incorpora nuevas vertientes de estudio, resultantes de su propia investigación o de lo compartido con colegas, estudiantes y tesistas. Así, nosotros aprendimos de ella, y me atrevo a pensar que, al acompañarnos en nuestras propuestas u "ocurrencias" temáticas y de investigación, ella aprendía con nosotros.

Brígida nos enseñó una forma peculiar de mirar el mundo familiar vinculado al trabajo, una perspectiva sociodemográfica que proviene de la mancuerna realizada entre la demografía y la sociología. Parte de esta mira-

* Profesora-investigadora de El Colegio de México, A.C., Centro de Estudios Demográficos, Urbanos y Ambientales. Correo: jnajera@colmex.mx ORCID: https://orcid.org/00000003-1995-0578 
da fue desarrollada en colaboración y compañía de Orlandina de Oliveira, profesora-investigadora del Centro de Estudios Sociológicos (CES). Por cierto, me disculpo por las veces que los estudiantes nos equivocábamos al escribir correctamente la referencia bibliográfica "García y Oliveira" u "Oliveira y García"; para nosotros, ustedes son un gran dúo.

Los estudiantes aprendimos de Brígida su forma de observar el mundo, no sólo en las clases, sino a través de la lectura de sus numerosos artículos, capítulos y libros; cuando ya no los encontrábamos a la venta, sentíamos tristeza y no quedaba más que "fotocopiar y engargolar", o ahora, "escanear y subirlos a la nube". Por todo esto, querida Brígida, permíteme decir, a manera de una portavoz, que más allá de las clases y de los cientos de textos que has escrito y hemos leído, lo que más valoramos los que fuimos tus alumnos es el acompañamiento, cariño, solidaridad y apoyo que nos brindaste, así como el seguimiento puntual y la vigilancia teórico-metodológica rigurosa a nuestras investigaciones; pero sobre todo, te agradecemos las largas horas de trabajo que nos dedicaste para lograr una buena investigación.

A lo largo de estos cuarenta años, la enseñanza de la perspectiva sociodemográfica ha sido un aporte por el que estudiantes y colegas hemos buscado a Brígida como profesora, directora de tesis, o miembro de comités académicos. Por ello, Brígida ha acompañado, como directora o lectora de tesis, a más de sesenta tesis de posgrado en El Colegio de México y en otras instituciones.

Ha dirigido tesis en los temas más clásicos, como fuerza de trabajo, hasta los más recientes, como uso del tiempo, trabajo no remunerado y trabajo de cuidados de adultos mayores. En este transitar figuran temas como población rural y migración temporal, organización social del trabajo, heterogeneidad laboral, participación económica femenina y juvenil, trabajadores asalariados y por cuenta propia, sector informal, trabajo decente, inestabilidad y segregación laboral, desempleo, paternidad y organización de la vida familiar, mercados de trabajo y desigualdades, condiciones laborales de poblaciones migrantes, violencia hacia las mujeres en ámbitos laborales, trayectorias a la vida adulta y trabajo de cuidados. Diversas generaciones de demógrafos y estudiosos de la población que convivimos con Brígida hemos sido beneficiados por la oportunidad de estudiar temas que en ese momento estaban ausentes, eran de vanguardia o de constante preocupación histórica.

Las enseñanzas de Brígida traspasaron las aulas para motivar a sus estudiantes a su primera incursión en la presentación de trabajos de investigación en espacios como la Reunión Nacional de la Sociedad Mexicana de Demografía, ALAP, IUSSP o PAA. Nos alentó a escribir el primer artículo o capítulo para su publicación, y algunos pudimos realizar proyectos edito- 
riales con ella. En estas formas variadas de docencia y difusión de la ciencia, nos enseñó a ser críticos, respetar y reconocer el saber acumulado, a indagar acerca de temas pertinentes para la sociedad, pero sobre todo, a mantener el rigor científico.

Sus alumnos, ahora profesores, investigadores o profesionistas, que laboran en universidades de este país o en el extranjero, en la función pública o en organismos internacionales, hemos llevado, transmitido y mostrado una forma de entender y explicar el vínculo trabajo y familia desde una perspectiva sociodemográfica. Por ello, sin duda alguna, ha sido una formadora de formadores, ya que hemos retransmitido sus enseñanzas y pensamiento sociodemográfico a otros.

La comunidad de demógrafos en el país, e incluso me atrevería a decir que la de América Latina, es pequeña, diversa y todos nos conocemos; pero algunos hemos sido emparentados por lazos académicos creados desde su persona, así que resulta de lo más satisfactorio sabernos herederos de sus enseñanzas. Gracias por haber sido y seguir siendo nuestra profesora.

Me resta señalar que la destacada trayectoria académica de la doctora Brígida García - de la cual he tenido el privilegio de ser parte, como su estudiante, tesista de posgrado y actualmente colega en el CEDUA- ha estado acompañada por su generosidad para compartir el conocimiento, siempre de manera entusiasta, en proyectos de investigación con estudiantes, tesistas, egresados y colegas de diversas instituciones, razón por la que estudiantes y colegas reconocemos la labor académica de Brígida en diversos espacios.

Hoy, ser reconocida como Profesora-Investigadora Emérita de El Colegio de México es un distintivo a su largo andar en la docencia, investigación y compromiso institucional. Reconocemos su amplia obra académica en México y Latinoamérica, su papel como formadora y precursora del pensamiento sociodemográfico, así como la trascendencia de éste en la política pública nacional e internacional, contribuyendo no sólo al desarrollo de las ciencias sociales, sino también como recurso para la reflexión de los grandes problemas sociodemográficos y laborales de México.

Querida Brígida, siéntete orgullosa del legado que has dejado en la academia, de la escuela que formaste, de tu aporte a la investigación, pero sobre todo, del gran ser humano que eres. Gracias por permitirnos ser parte de tu historia sociodemográfica. 


\section{Palabras de Brígida García Guzmán*}

Estimados integrantes del presídium, funcionarios de El Colegio de México, colegas, alumnos, amigas y amigos:

Me honra de manera especial recibir la distinción de Profesora-Investigadora Emérita de El Colegio de México, y en esta ocasión me gustaría compartir con ustedes un breve recuento de mi experiencia como académica y de lo que he buscado contribuir en lo que atañe a la generación de conocimiento en el campo de la sociodemografía y en la formación de recursos humanos.

Llegué a El Colegio en 1969 desde República Dominicana para estudiar la maestría en Demografía, una disciplina hasta ese entonces bastante desconocida para mí. Tuve la fortuna de integrarme a la planta docente al terminar la maestría, ya que se necesitaban investigadores para analizar la recién recolectada Encuesta de Fecundidad Rural, la primera realizada en el país en ese ámbito territorial.

Aunque escribí algunos trabajos e impartí clases sobre fecundidad con Beatriz Figueroa, poco a poco me fui especializando en el estudio del trabajo en sus múltiples facetas, así como en las repercusiones de la participación laboral sobre el bienestar de las personas, tanto hombres como mujeres. Considero que mis investigaciones han contribuido a entender el impacto del cambio en la estrategia económica de México sobre los mercados de trabajo y las familias, así como las interrelaciones entre las transformaciones demográficas y el estatus de las mujeres.

\section{Mercados laborales}

Mis estudios sobre los mercados laborales se han encaminado en tres direcciones principales: 1) conocer la evolución de los distintos sectores de trabajadores asalariados y no asalariados (por cuenta propia) en actividades industriales o de servicios en diferentes momentos históricos, así como las condiciones de trabajo que enfrentan; 2) cuestionar y proponer alternativas en torno a las categorías de análisis más frecuentemente utilizadas para explicar las carencias laborales del país; 3) precisar los factores intervinientes en el aumento de la actividad económica femenina, pues se trata del grupo poblacional en el cual se han observado las mayores transformaciones. En

* Profesora-Investigadora Emérita de El Colegio de México, A.C. Correo: bgarcia@ colmex.mx ORCID: http://orcid.org/0000-0003-2223-5225 
estas investigaciones no sólo he enfrentado problemas teóricos y metodológicos, sino dificultades técnicas para compatibilizar fuentes de información a lo largo de muchos años, particularmente cuando he buscado profundizar en lo ocurrido en el ámbito regional o local.

Un proceso al cual le he prestado particular atención ha sido la salarización o asalarización de la mano de obra en el país, dado que el sistema capitalista prevaleciente descansa principalmente en la compraventa de fuerza de trabajo. En desarrollos teóricos de diversa índole se ha postulado la hipótesis de que, una vez en marcha, este proceso abarca de manera inexorable a la totalidad de la fuerza de trabajo. No obstante, existe cada vez mayor evidencia, sobre todo en los países no desarrollados, de que la salarización de la mano de obra no tiene lugar de la misma forma que se dio en los países occidentales desarrollados. El aporte que he buscado hacer ha consistido en precisar los momentos más importantes en la expansión de este proceso en distintas regiones del país, así como sustentar, con la mejor información y herramientas estadísticas posibles, su estancamiento ya en la década de 1970 . He podido demostrar que es en las regiones de mayor dinamismo económico, como es el caso de la Ciudad de México, donde se reproducen los sectores no asalariados con mayor celeridad. En estos casos es posible pensar en la retroalimentación de los sectores asalariados y no asalariados mediante fenómenos como la subcontratación industrial, comercial o de servicios. Asimismo, no habría que olvidar que la mayor concentración poblacional en estas zonas más dinámicas seguramente influye para que también allí proliferen los trabajadores no asalariados más desprotegidos.

Otro tipo de interés en lo que respecta al campo de la fuerza de trabajo ha sido mi búsqueda constante por satisfacer -mediante mis escritos, mi actividad docente y las direcciones de tesis, así como por medio de mis contactos con productores de información nacional (INEGI, principalmente)la necesidad que tenemos de lograr mayor claridad conceptual en la definición de indicadores sobre ocupación, empleo y desempleo en México. Muchas veces he sido testigo de cómo los conceptos generados por organismos internacionales, las corrientes científicas que se consideran más novedosas, el financiamiento existente, así como la fuerza o debilidad del personal encargado de poner en práctica relevantes procesos de generación de información (censos poblacionales, encuestas continuas de empleo y demográficas) influyen de manera considerable en el conocimiento que se genera. Me he concentrado en dejar claros los ejes comunes (y las diferencias) entre conceptos tales como marginalidad, informalidad, precariedad, trabajo decente, y en hacer visible la necesidad de comprender qué es lo que se busca medir 
como un paso necesario para la creación de indicadores comprensibles para los no especialistas y los encargados de elaborar políticas.

Finalmente, en esta área no podría dejar de subrayar mi compromiso por estimular la generación de conocimiento en torno a las implicaciones demográficas de la participación económica femenina, tanto en los países desarrollados como en los no desarrollados; es decir, la vinculación de este fenómeno económico con la formación y la disolución familiar, la migración, la salud y el comportamiento reproductivo.

\section{Familias y hogares}

Ahora bien, la oferta de mano de obra no está constituida por individuos aislados, sino por personas que forman parte de hogares o familias, los cuales pueden facilitar o constreñir la participación individual en el mercado de trabajo. Esta premisa, así como el cambio en la unidad de análisis que trae consigo, ha permitido enriquecer el conocimiento existente sobre la dinámica de los mercados laborales y la manera en que se vive y reproduce la desigualdad en países como los nuestros. Asimismo, la división del trabajo remunerado y no remunerado entre hombres y mujeres en el interior de las unidades domésticas, así como las formas de convivencia, constituyen objetos de estudio en sí mismos de suma relevancia en una sociedad como la mexicana, con lazos familiares muy fuertes. He formado parte de equipos de investigación pioneros en México en la investigación de estos fenómenos y relaciones -inicialmente con Orlandina de Oliveira y Humberto Muñoz, y más recientemente con Edith Pacheco y también con Jéssica Nájera-. De esta manera, hemos podido profundizar en el conocimiento del proceso mediante el cual se reproduce la desigualdad y se sobrevive cotidianamente en situaciones de precariedad y pobreza. Vivir en un mismo hogar significa compartir algunos beneficios derivados de las condiciones económicas de los demás miembros, pero para la gran mayoría de la población lo que sucede es que así se busca compensar los bajos ingresos de unos con los bajos ingresos de los otros.

Acerca del estudio de las familias o unidades domésticas, he desarrollado dos investigaciones de largo aliento que, desde mi perspectiva, merecen una consideración especial. Los resultados de la primera se encuentran en el libro Trabajo femenino y vida familiar en México, publicado en 1994 (y reimpreso unos años después) por El Colegio de México. Este libro, elaborado conjuntamente con Orlandina, ha sido el de mayor impacto entre mis publicaciones, y esto probablemente se debe a que allí se documenta de 
manera precisa el crecimiento de la participación laboral femenina en México, especialmente durante la crisis económica de los años 1980, y se fundamenta el importante papel económico que han tenido desde entonces las mujeres de más edad, unidas y con hijos. Asimismo, en el libro se explora el significado del trabajo extradoméstico para las mujeres, el cual la mayoría de las veces se ejerce en función de las necesidades familiares; es decir, encontramos que en pocas ocasiones las mujeres mexicanas hacen alusión a su participación económica en términos personales, diferenciados de sus roles maternos.

Como es bien conocido, los estudios cualitativos abren un sinnúmero de pistas de investigación, y Orlandina y yo seguimos algunas de ellas mediante una investigación posterior sobre la dinámica familiar en la Ciudad de México y Monterrey, basada en una encuesta probabilística. Dicha encuesta constituyó una experiencia relevante desde numerosos puntos de vista, pues allí diseñamos los cuestionarios, aunque la configuración de las muestras y la recolección de la información estuvieron a cargo del INEGI. Lo novedoso fue incluir ahora a los varones, además de las mujeres, y analizar tres dimensiones de la vida familiar: la división del trabajo extradoméstico y doméstico, las formas de convivencia (patrones de autoridad, libertad de movimiento y violencia doméstica), y las concepciones sobre los roles considerados como adecuados para hombres y mujeres.

Las reflexiones anteriores me llevan a subrayar mi involucramiento en el área de población y género. Muchas veces se ha sostenido que un cambio en el estatus de las mujeres (o un empoderamiento femenino) podría coadyuvar en la reducción del crecimiento poblacional, así como contribuir a la salud infantil, entre otros resultados sociodemográficos deseables. Se trata de planteamientos controvertidos, ya que el mejoramiento de la condición femenina es una meta en sí misma y así ha sido reconocido por académicos, políticos y activistas por igual. Sin embargo, un efecto importante de la consideración de estas grandes metas en sentido conjunto, ha sido la ampliación de las investigaciones científicas y las fuentes de financiamiento para estudios sobre mujeres, género y población. En este contexto, he podido convocar a investigadores e investigadoras nacionales y extranjeros para realizar reflexiones que buscan ampliar el conocimiento existente mediante publicaciones conjuntas. Estas convocatorias han sido facilitadas por mi labor en asociaciones académicas como la Sociedad Mexicana de Demografía y la Unión Internacional para el Estudio Científico de la Población.

Mis intereses de investigación más recientes giran en torno al trabajo no remunerado, esto es, al trabajo doméstico y de cuidado. La mayor dedicación de las mujeres al trabajo no remunerado, así como su valor económico, ha 
sido ya evidenciado de diversas maneras y la información recolectada en el país sobre este trabajo es muy nutrida. Con estos estudios hemos procurado respaldar o refutar diversos planteamientos sobre las desigualdades sociales y de género en el uso del tiempo, así como señalar los ámbitos donde se visualizan trazos de transformación y las tendencias que permanecen.

Lo dicho hasta aquí se refiere a la investigación. Sin embargo, este breve recuento quedaría muy incompleto si no compartiera con ustedes algunas pinceladas sobre mi actividad docente. He tenido la fortuna de poder impartir clases sobre mis temas de investigación: fecundidad, fuerza de trabajo, teoría y metodología en los estudios de población, principalmente. Por medio de mis cursos he podido conocer a un sinnúmero de personas maravillosas que han sido mis estudiantes, muchos aquí presentes. Ha sido muy significativo verlos crecer y desarrollarse. Cada vez que inicio un curso se trata de una verdadera aventura intelectual en la cual siempre aplico el principio de que todos los alumnos van a aprender siempre que logre transmitir bien mis ideas y planteamientos, y ellos por supuesto contribuyan con su esfuerzo. Lo único que va a variar es el tiempo involucrado. De esta manera he sido testigo privilegiado de la conformación de la comunidad demográfica en el país y en América Latina.

Finalmente, ha llegado la hora de los agradecimientos. Esta parte siempre me preocupa por la posibilidad de los olvidos, pero si así ocurriese, apelo desde ya a su comprensión por utilizar la forma colectiva. Reconozco las oportunidades que me ha abierto esta institución en mi vida profesional, y quiero expresar mi agradecimiento a sus autoridades de muchos años y a los funcionarios que han hecho posible mi labor de investigación y docencia. Es muy significativo para mí este nombramiento, y le doy las gracias a la actual presidenta, Silvia Giorguli, por haberlo impulsado, y a la Junta de Gobierno, al Consejo Académico y a las colegas que lo apoyaron de manera particular.

A mis hijos Alicia Acely, Ernesto Rafael y Mark, les recuerdo que no olvido su respaldo y solidaridad, y a Gustavo, mi esposo y colega durante ya más de cuatro décadas, le expreso mi reconocimiento por su apoyo incondicional. A todos los aquí presentes, compañeros, amigas y amigos, gracias por acompañarme. Un fuerte abrazo a todos ustedes. 
